\title{
Phase-change hybrids for thermo-responsive sensors and actuators
}

\author{
Young-Jae Jin ${ }^{1}$, Beomsu Shin-Il Kim¹, Wang-Eun Lee ${ }^{2}$, Chang-Lyoul Lee ${ }^{3}$, Hyojin Kim ${ }^{4}$, Kyu-Ho Song ${ }^{4}$, \\ Sung-Yeon Jang ${ }^{5}$ and Giseop Kwak ${ }^{1}$
}

Highly advanced phase-change hybrids ( $\mathrm{PCHs}$ ), which consist of a phase-change material and conjugated polymer, were developed for new sensor and actuator applications. PCH films with excellent characteristics were obtained simply by depositing various molten paraffin waxes (PWs) in situ onto poly(diphenylacetylene) (PDPA) films with extremely large fractional free volumes. The phase-change enthalpy of the PWs in the hybrid films was quite high and remained constant over prolonged use. The PCH films underwent critical changes in both fluorescence (FL) intensity and color during the phase change of the PWs, which facilitated various sensor applications such as highly reversible writing/erasing, fingerprinting and array-type thermometer usage. In addition, a biaxially oriented polypropylene (BOPP)-supported PCH film exhibited extremely fast and highly reproducible thermomechanical actuation with reversible curling/uncurling during the phase change of the PWs. These findings will be useful for developing novel $\mathrm{PCH}$ materials with highly advanced functions and applications.

NPG Asia Materials (2014) 6, e137; doi:10.1038/am.2014.94; published online 10 October 2014

\section{INTRODUCTION}

Phase-change materials (PCMs) have recently attracted considerable attention because of their ability to reversibly store and release large amounts of latent heat, which can be applied for the thermal energy management of natural heat sources and waste heat. ${ }^{1-5}$ For practical applications, PCMs have often been incorporated in organic or inorganic matrices to enhance their mechanical integrity and cyclic stability on repeated use. The matrix material can also affect the phasechange properties of the resultant PCM-based composites, and some of these composites have exhibited highly enhanced phase-change enthalpy and thermal conductivity. ${ }^{6-11}$ Additionally, actuators based on PCMs have been developed for other purposes. ${ }^{12-14}$ To the best of our knowledge, however, there are no reports on PCMs that can exhibit both optical output signals and thermomechanical actuation in the heat storage/release process. Such sensory and actuation functions would make it possible to monitor the latent heat in real time and further enhance thermal energy management processes. To achieve such intelligent functions in PCMs, the matrices should be designed such that the optical and thermomechanical properties readily vary at the transition temperature. The matrices should also be impregnated with PCMs and accommodate the expected thermodynamic volume change without leakage for a long period. Thus, the matrix materials should have a porous structure.

Conjugated polymers (CPs) respond promptly to changes in their electronic structure, even to minor changes, because of their intrinsic conjugation cooperativity. ${ }^{15-19}$ Their optical properties can significantly change in response to molecular perturbations driven by external chemical or physical stimuli. Therefore, from the viewpoint of optical sensor applications, CPs can be considered potential candidates for intelligent organic matrices. However, conventional CPs typically have planar geometries with strong intermolecular interactions because of their stiff and rigid main chains, ${ }^{20-25}$ which results in a highly dense chain packing structure in the solid-state. Therefore, the use of such crystalline CPs as porous matrices for impregnation with PCMs is restricted.

Unlike conventional CPs, poly(diphenylacetylene) (PDPA) derivatives contain a large fractional free volume with numerous molecularscale microvoids in the bulk solid state because of their intrinsic amorphous nature. ${ }^{26,27}$ Various chemicals such as hydrocarbons, alcohols and even silicon oils can readily diffuse into bulk PDPAs under ambient conditions. ${ }^{28-32}$ Notably, the intramolecular stack structure of the side-group phenyl rings in PDPAs is fully relaxed in the swollen state, and consequently, the fluorescence (FL) emission of

${ }^{1}$ School of Applied Chemical Engineering, Major in Polymer Science and Engineering, Kyungpook National University 1370 Sankyuk-dong, Buk-ku, Daegu, Korea; ${ }^{2}$ Reliability Assessment Center for Chemical Materials, Korea Research Institute of Chemical Technology(KRICT), 141 Gajeong-ro, Yuseong-gu, Daejeon, Korea; ${ }^{3}$ Advanced Photonics Research Institute, Gwangju Institute of Science and Technology, 1 Oryong-dong, Buk-gu, Gwangju, Korea; ${ }^{4}$ Daegu Technopark Nano Convergence Practical Application Center, 891-5 Daecheon-dong, Dalseo-ku, Daegu, Korea and ${ }^{5}$ Department of Chemistry, Kookmin University, 861-1 Jeongneung-dong, Seongbuk-gu, Seoul, Korea

Correspondence: Professor S-Y Jang, Institution: Kookmin University, Department of Chemistry, Kookmin University, 861-1 Jeongneung-dong, Seongbuk-gu, Seoul 136-702, Korea.

E-mail: syjang@kookmin.ac.kr

or Professor G Kwak, Institution: Kyungpook National University, School of Applied Chemical Engineering, Major in Polymer Science and Engineering, Kyungpook National University 1370 Sankyuk-dong, Buk-ku, Daegu 702-701, Korea.

E-mail: gkwak@knu.ac.kr

Received 27 June 2014; revised 11 August 2014; accepted 19 August 2014 
PDPAs can be strongly enhanced. ${ }^{33-36}$ Furthermore, the degree of FL increase differs significantly based on the viscosity of the diffused medium because the degree of torsion angle relaxation of the side phenyl rings is affected by the friction energy transferred from the medium to these phenyl rings. ${ }^{37}$ Accordingly, PDPA derivatives would be potential porous organic matrices for PCMs and also exhibit an optical sensory response, as required in the present work.

Thermomechanical actuation of soft materials is generally based on a reversible thermodynamic volume change due to phase transition or thermoisomerization in the bulk solid state, ${ }^{38-43}$ whereas the optical change of CPs is primarily caused by variations in the electronic structure. Therefore, if the PDPA matrix is infiltrated with a certain PCM, and the polymer matrix undergoes further changes in the volume and electronic structure simultaneously during the phase change of the PCM, the PCM-incorporated PDPA film can act as a unique multifunctional hybrid material that exhibits both optical and thermomechanical responses to latent heat.

In this study, we developed phase-change hybrids ( $\mathrm{PCHs}$ ) by infiltrating PDPA films with paraffin waxes (PWs). The resultant $\mathrm{PCH}$ films remained stable without leakage of the PW during repeated phase-change tests. The phase-change enthalpy of the PW in the PDPA films was relatively high and remained constant for long periods of use. The FL emission of the $\mathrm{PCH}$ films varied in a systematic manner over the phase change. In addition to the optical response, the $\mathrm{PCH}$ films exhibited considerable mechanical deformation upon reversible heating and cooling. These multifunctional responses (FL change and thermomechanical actuation) were extremely fast, and a high degree of reproducibility was achieved without any sign of mechanical fatigue. Herein we describe the details of the preparation, thermal storage/release capacity, FL response and thermomechanical actuation behavior of the $\mathrm{PCH}$ films. In addition, we suggest new applications for our PCHs involving their potential use as sensors and actuators.

\section{MATERIALS AND METHODS}

\section{Materials}

$\mathrm{PDPA}_{\mathrm{Cl}}$ and $\mathrm{PDPA}_{\mathrm{C} 18}$ were synthesized using a methodology reported elsewhere. ${ }^{44,45}$ The PDPAs used in this study had high average molecular weights $\left(M_{w}\right)$ of $1.34 \times 10^{6}$ and $8.7 \times 10^{6} \mathrm{~g} \mathrm{~mol}^{-1}$ and polydispersity indices $\left(\mathrm{PDI}=M_{w} / M_{n}\right)$ of 1.2 and 1.4, respectively. The poly[2-(2'-ethylhexyloxy)-5methoxy-1,4-phenyl enevinylene] was purchased from Sigma-Aldrich Co. Ltd. (St Louis, MO, USA). The PWs were purchased from TCI (Tokyo Chemical Industry Co. Ltd., Tokyo, Japan). The biaxially oriented polypropylene (BOPP) film material, which is often used for cigarette packaging, was purchased from KT\&G (Korea Tobacco \& Ginseng Corp., Daejeon, Korea).

\section{Preparation of $\mathrm{PW}-\mathrm{PDPA}_{\mathrm{C} 1}$ hybrid films}

$\mathrm{PDPA}_{\mathrm{Cl}}$ was dissolved in toluene (concentration $\approx 2.0 \mathrm{wt} \%$ ) and cast onto appropriate substrates. Then, bulk liquid PWs were deposited onto the PDPA $\mathrm{Cl}$

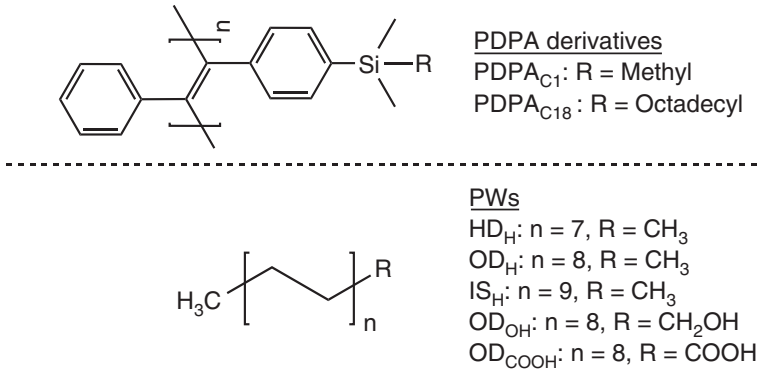

Figure 1 Chemical structures of PDPA derivatives and PWs. film (size: $30 \mathrm{~mm}$ (radius) $\times 100 \mu \mathrm{m}$ (thickness)) without dilution at temperatures of $5{ }^{\circ} \mathrm{C}$ above $T_{m}$. The excess PW residues were removed by spinning the film at a high speed (greater than $2000 \mathrm{rpm}$ ) followed by blotting using oil adsorption pads (Yuhan Kimberly, Ltd., Seoul, Korea).

\section{Preparation of $\mathrm{OD}_{\mathrm{H}}-\mathrm{PDPA}_{\mathrm{C} 1} / \mathrm{BOPP}$ bilayer films}

$\mathrm{PDPA}_{\mathrm{Cl}}$ was dissolved in toluene (concentration $\approx 1.0 \mathrm{wt} \%$ ) and cast on top of the BOPP film ( $15 \mathrm{~mm}$ (length) $\times 5 \mathrm{~mm}$ (width) $\times 20 \mu \mathrm{m}$ (thickness)). The deposition of $\mathrm{OD}_{\mathrm{H}}$ onto the $\mathrm{PDPA}_{\mathrm{Cl}}$ layer was performed using the same procedure described above.

\section{Measurements}

The FL emission spectra were recorded on a FP-6500 spectrofluorometer (Jasco, Tokyo, Japan) at an excitation wavelength of $420 \mathrm{~nm}$ and a scanning rate of $100 \mathrm{~nm} \mathrm{~min}^{-1}$ at room temperature. The temperature-variable FL emission spectra and plot were recorded on the same spectrofluorometer equipped with an ETC-273 temperature controller (Jasco) at a scanning rate of $10000 \mathrm{~nm}$ $\mathrm{min}^{-1}$. Both the heating and cooling rates were set to $20^{\circ} \mathrm{C} \mathrm{min}^{-1}$. Ultraviolet absorption spectroscopy was performed on a $\mathrm{V}-650$ spectrophotometer (Jasco). Fourier transform infrared (FT-IR) spectra were recorded on a FT/IR 4100 spectrometer (Jasco) equipped with an attenuated total reflectance (ATR, PRO450-S, Jasco). Fluorescence images and videos were recorded with a digital camera (PowerShot A2000 IS, Canon, Tokyo, Japan) under a ultraviolet lamp with an excitation wavelength of $>365 \mathrm{~nm}$. X-ray diffraction (PANalytical X'Pert PRO-MPD, PANalytical, Almelo, Netherlands) was performed at room temperature at the Korea Basic Science Institute (Daegu, South Korea). The samples were mounted directly onto the diffractometer. The experiment was performed using $\mathrm{CuK} \alpha(1.54 \AA)$ radiation at $40 \mathrm{kV}$ and $25 \mathrm{~mA}$. Differentia scanning calorimetry (DSC; DSC141 evo, SETARAM Instrumentation, Caluire, France) was performed under pure nitrogen gas at heating and cooling rates of $10^{\circ} \mathrm{C} \mathrm{min}^{-1}$. TGA (Labsys-evo, SETARAM Instrumentation) was performed under pure nitrogen gas at a heating rate of $10^{\circ} \mathrm{C} \mathrm{min}^{-1}$. Cross-sectional FL images were recorded on an Eclipse E600 fluorescence optical microscope (Nikon, Tokyo, Japan) equipped with a DS Fil digital camera (Nikon) and a super-high-pressure 100-W Hg lamp (HBO103W3/2, OSRAM, Munich, Germany). The scanning electron microscope images were taken at room temperature using a field-emission scanning electron microscope (S-4300, Hitachi, Ltd., Tokyo, Japan). For the scanning electron microscope analysis, the samples were sputter-coated with gold.

\section{RESULTS}

The ultimate goal of this study was to develop PW - PDPA hybrid films with advanced functionality during the heat storage/release process. From many PDPA derivatives, poly[1-phenyl-2-(p-trimethylsilyl) phenylacetylene] $\left(\mathrm{PDPA}_{\mathrm{Cl}}\right.$ in Figure 1) was selected as the matrix because this material is a strongly fluorescent polymer with an extremely large fractional free volume $(>0.26)^{27}$ (BET surface area: $635 \mathrm{~m}^{2} \mathrm{~g}^{-1}$; total pore volume: $\left.0.36 \mathrm{~cm}^{3} \mathrm{~g}^{-1}\right) .{ }^{46}$ The PDPA $\mathrm{C} 1$ used in this study had a relatively high average molecular weight $\left(M_{w}=1.34 \times 10^{6} \mathrm{~g} \mathrm{~mol}^{-1}\right)$ with a narrow polydispersity index $(\mathrm{PDI}=$ $M_{w} / M_{n}=1.2$ ).

Three types of PWs were used in the present study (Figure 1): (i) hydrocarbon-type PWs: hexadecane $\left(\mathrm{HD}_{\mathrm{H}}\right.$ : melting temperature, $T_{m}: 22.8^{\circ} \mathrm{C}$; enthalpy of fusion, $\left.\Delta H_{f u s}: 184.8 \mathrm{~J} \mathrm{~g}^{-1}\right)$; octadecane $\left(\mathrm{OD}_{\mathrm{H}}\right.$ : $T_{m}: 32.0^{\circ} \mathrm{C} ; \Delta H_{\text {fus }}: 192.4 \mathrm{~J} \mathrm{~g}^{-1}$ ) and icosane (IS $\mathrm{I}_{\mathrm{H}}: T_{m}: 40.9^{\circ} \mathrm{C} ; \Delta H_{\text {fus }}:$ $\left.204.1 \mathrm{~J} \mathrm{~g}^{-1}\right)$; (ii) an alcohol-type PW: octadecanol ( $\mathrm{OD}_{\mathrm{OH}}: T_{m}: 61.5^{\circ} \mathrm{C}$; $\Delta H_{\text {fus }}: 193.3 \mathrm{~J} \mathrm{~g}^{-1}$ ); and (iii) an acid-type PW: octadecanoic acid (OD $\mathrm{COOH}: T_{m}: 73.0^{\circ} \mathrm{C} ; \Delta H_{\text {fus }}: 175.2 \mathrm{~J} \mathrm{~g}^{-1}$ ). All of the values reported here were measured by our group and differ only slightly from the standard reference values (Table 1). ${ }^{47,48}$

The PCHs were prepared by the in situ infiltration of $\mathrm{PDPA}_{\mathrm{C} 1}$ films (thickness $\approx 10 \mu \mathrm{m}$, prepared by solvent casting on a $30-\mu \mathrm{m}$-thick glass substrate) with PWs. Molten liquid-state PWs at $\sim 5^{\circ} \mathrm{C}$ above their melting temperature $\left(T_{m}\right)$ were deposited onto the thick $\mathrm{PDPA}_{\mathrm{C} 1}$ 
Table 1 Thermodynamic properties of pure PWs and hybrid films

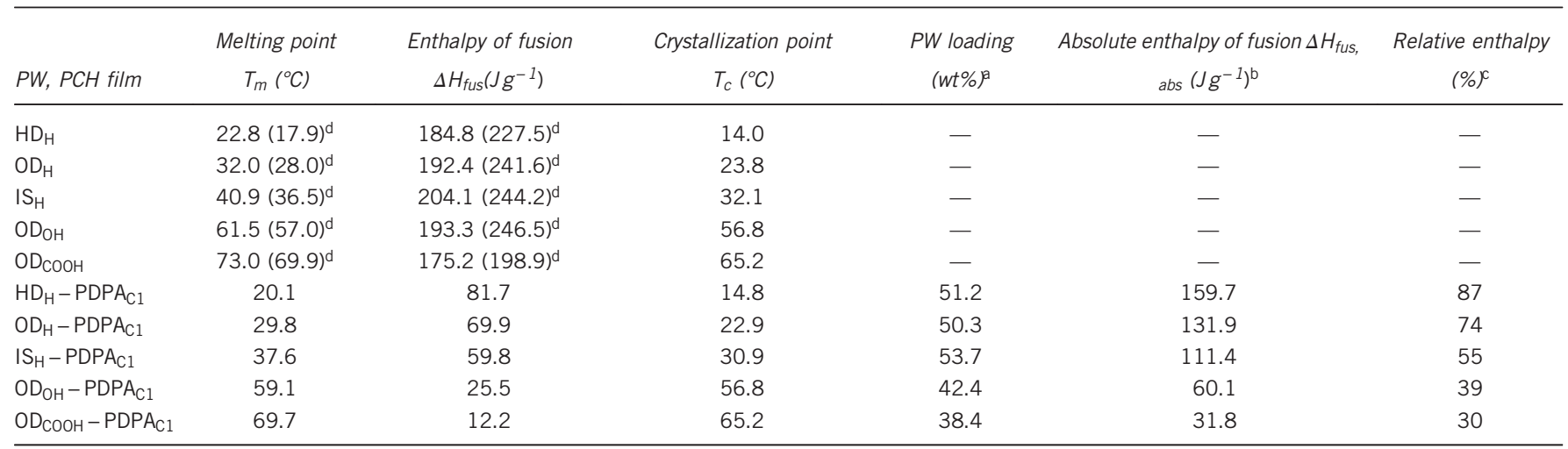

Abbreviations: $\mathrm{HD}_{\mathrm{H}}$, hexadecane; $\mathrm{IS}_{\mathrm{H}}$, icosane; $\mathrm{OD}_{\mathrm{COOH}}$, octadecanoic acid; $\mathrm{OD}_{\mathrm{H}}$, octadecane; $\mathrm{OD}_{\mathrm{OH}}$, octadecanol; PCH, phase-change hybrid; PDPA, poly(diphenylacetylene); PW, paraffin wax; $T_{c}$, temperature of crystallization; $T_{m}$, melting temperature.

aData from TGA.

betermined by dividing the apparent enthalpy $\left(\Delta H_{\text {fus }}\right.$, including the mass of PDPA $\left.A_{C 1}\right)$ by the PW loading.

${ }^{c}\left(\Delta H_{\text {fus, abs }}\right.$ of hybrid film $\left.\times 100\right) / \Delta H_{\text {fus }}$ of the corresponding pure PW.

dReference values. ${ }^{42,43}$

a

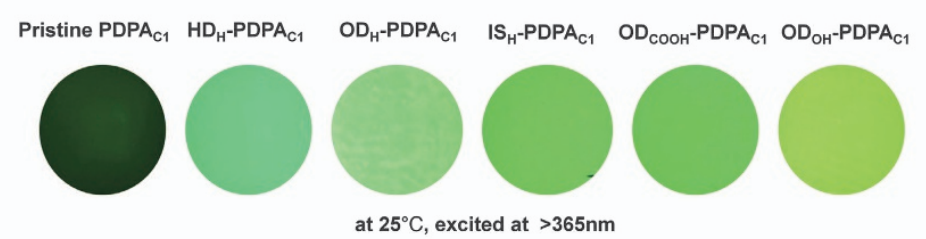

C

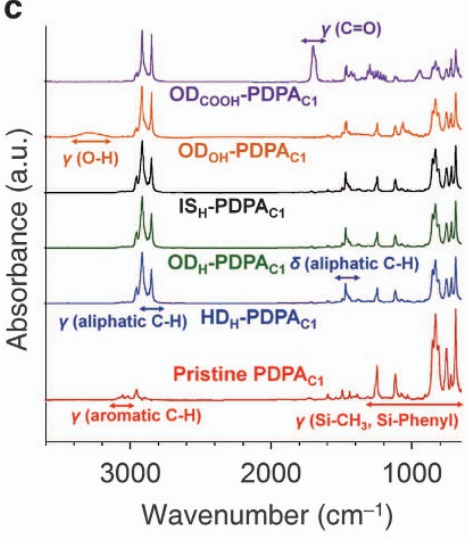

d

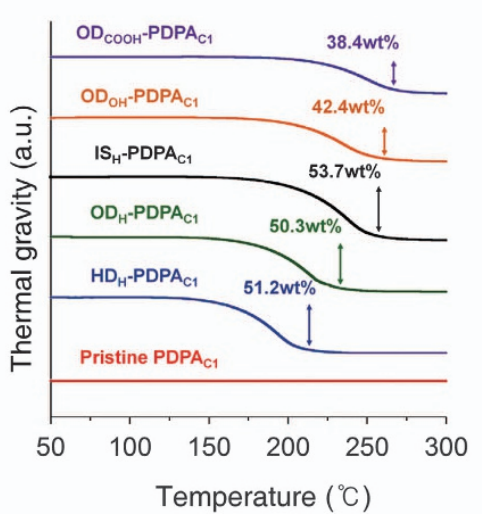

b

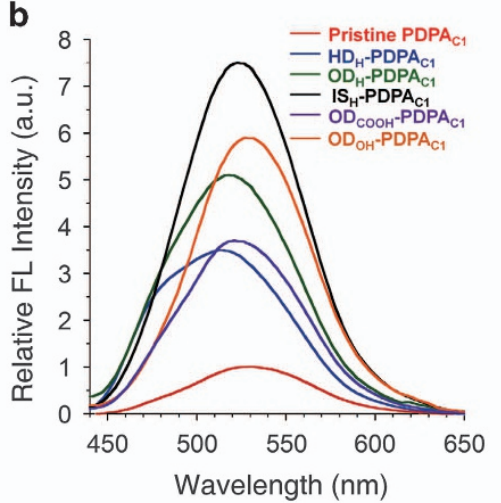

e

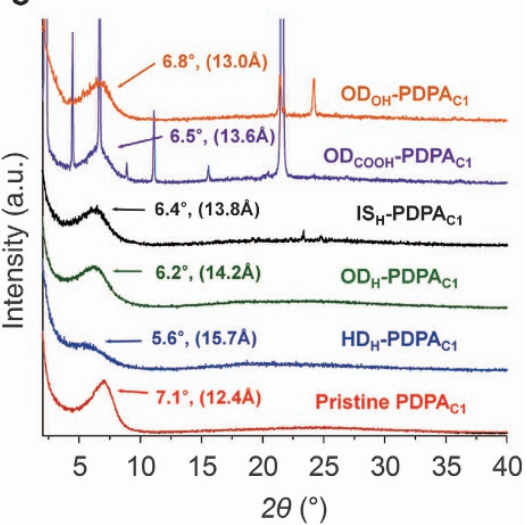

Figure 2 Characterization of PCH films. (a) FL images, (b) FL emission spectra (excited at $420 \mathrm{~nm}$ ), (c) FT-IR spectra, (d) TGA thermograms and (e) X-ray diffraction patterns of $\mathrm{PCH}$ films. The pristine PDPA $\mathrm{Cl}_{\mathrm{f}}$ film (thickness $\approx 10 \mu \mathrm{m}$ ) in (a) was prepared using the solvent casting method. The relative intensity in (b) is $I_{\text {hybrid }} I_{\text {pristine, }}$ where $I_{\text {hybrid }}$ is the FL intensity of the hybrid film and $I_{\text {pristine }}$ is the corresponding value for the pristine film.

films. The PWs readily diffused into the films through internal microvoids, as was confirmed by the immediate swelling of the films. Excess PW residues were removed by spinning the films at speeds greater than 2000 r.p.m. The resultant $\mathrm{PCH}$ films containing various $\mathrm{PWs}$ in $\mathrm{PDPA}_{\mathrm{C} 1}$ films are shown in Figure $2 \mathrm{a}$. The $\mathrm{FL}$ emission intensity of our PDPA ${ }_{C 1}$ film increased noticeably after PW infiltration, whereas the maximum intensity wavelength of FL emission $\left(\lambda_{\max }\right.$, ${ }_{F L}$ ) was blue-shifted (Figure $2 \mathrm{~b}$ ). For $\mathrm{OD}_{\mathrm{H}}-\mathrm{PDPA}_{\mathrm{Cl}}$, the FL intensity was $\sim 5.1$ times higher than that of the pristine $\mathrm{PDPA}_{\mathrm{C} 1}$ film, and $\lambda_{\max }$, FL was blue-shifted by $15 \mathrm{~nm}(518 \mathrm{~nm})$ relative to the pristine film $(533 \mathrm{~nm}) . \mathrm{PDPA}_{\mathrm{C} 1}$ could accommodate a wide range of PWs that have different molecular weights and polarities. The observed FL enhancement is attributed to the degeneration of nonradiative emission decay channels of $\mathrm{PDPA}_{\mathrm{C} 1}$, which occurred because of the relaxation of intramolecular $\pi$-stacking on the side-group phenyl rings resulting from the plasticizer effect of $\mathrm{OD}_{\mathrm{H}} \cdot{ }^{33-36}$ However, the ultraviolet - 
visible absorption spectrum of the $\mathrm{OD}_{\mathrm{H}}-\mathrm{PDPA}_{\mathrm{C} 1}$ matched that of the pristine film, indicating that neither aggregation nor phase separation occurred during the impregnation (Supplementary Figure 1). Other PW - PDPAs also exhibited similar trends in their emission properties, with some dependence on the PW type (Figure $2 \mathrm{~b}$ ).

The FL emission properties of all the $\mathrm{PW}-\mathrm{PDPA}_{\mathrm{C} 1}$ materials studied are summarized in Supplementary Table 1 together with the properties of the pristine films for comparison. Notably, the relative FL intensity increased in the order $3.7\left(\mathrm{HD}_{\mathrm{H}}\right)<5.1\left(\mathrm{OD}_{\mathrm{H}}\right)<7.5\left(\mathrm{IS}_{\mathrm{H}}\right)$ as a function of the molecular weight of the PWs, whereas the blue shift of $\lambda_{\max , F L}$ decreased as $20\left(\mathrm{HD}_{\mathrm{H}}\right)>15\left(\mathrm{OD}_{\mathrm{H}}\right)>10\left(\mathrm{IS}_{\mathrm{H}}\right) \mathrm{nm}$. In larger PWs, molecular friction with the matrix $\mathrm{PDPA}_{\mathrm{C} 1}$ is weakened because the viscosity should be proportional to the molecular size. Moreover, as interfacial frictional forces between $\mathrm{PDPA}_{\mathrm{C} 1}$ and $\mathrm{PW}$ are suppressed, the nonradiative emission decay of the matrix $\mathrm{PDPA}_{\mathrm{Cl}}$ should degenerate because of the reduced colloidal quenching. However, for PWs with a low $T_{m}$, their higher molecular mobility can facilitate relaxation of the phenyl-phenyl stack structure, thus predominantly resulting in larger blue shifts. Both acid- and alcoholtype $\mathrm{PW}\left(\mathrm{OD}_{\mathrm{COOH}}, \mathrm{OD}_{\mathrm{OH}}\right)$-containing $\mathrm{PCH}$ films induced smaller blue shifts compared with the corresponding $\mathrm{OD}_{\mathrm{H}^{-}}$containing hybrid because heterologous PWs exhibit lower molecular mobility because of the much stronger intermolecular interactions, including hydrogen bonding. The blue shift of $\lambda_{\max , F L}$ decreased in the order $15\left(\mathrm{OD}_{\mathrm{H}}\right)$ $>12\left(\mathrm{OD}_{\mathrm{COOH}}\right)>6\left(\mathrm{OD}_{\mathrm{OH}}\right) \mathrm{nm}$ (Supplementary Table 1$)$. Note that we attempted to prepare PCHs via the in situ infiltration of poly[2-(2'ethylhexyloxy)-5-methoxy-1,4-phenyl enevinylene], which is a conventional crystalline CP, with PWs. Poly[2-(2'-ethylhexyloxy)-5methoxy-1,4-phenyl enevinylene] exhibited minimal swelling even on prolonged exposure to PWs, and there were no FL changes, confirming that the amorphous nature and porosity of our PDPA $\mathrm{C}_{1}$ are crucial factors for this material to serve as a matrix polymer for PCHs (Supplementary Figure 2).

Figure $2 \mathrm{c}$ presents the attenuated total reflectance mode FT-IR spectra of the $\mathrm{PCH}$ films. The $\mathrm{HD}_{\mathrm{H}}-, \mathrm{OD}_{\mathrm{H}}-$ and $\mathrm{IS}_{\mathrm{H}}-\mathrm{PDPA}_{\mathrm{Cl}}$ films exhibited relatively strong peaks characteristic of aliphatic hydrocarbon PWs at 2900 and $2840 \mathrm{~cm}^{-1}$, attributable to $\mathrm{C}-\mathrm{H}$ stretching, in addition to absorption peaks characteristic of the $\mathrm{Si}-\mathrm{CH}_{3}\left(1248,854 \mathrm{~cm}^{-1}\right)$ and $\mathrm{Si}-$ phenyl $\left(1118 \mathrm{~cm}^{-1}\right)$ groups of $\mathrm{PDPA}_{\mathrm{C} 1}$. The $\mathrm{OD}_{\mathrm{OH}}-$ and $\mathrm{OD}_{\mathrm{COOH}}-\mathrm{PDPA}_{\mathrm{C} 1}$ films displayed characteristic absorption peaks at $3317 \mathrm{~cm}^{-1}(\mathrm{O}-\mathrm{H}$ stretching) and $1697 \mathrm{~cm}^{-1}$ ( $\mathrm{C}=\mathrm{O}$ stretching), respectively, which further confirmed that all the PWs tested in our study effectively infiltrated the $\mathrm{PDPA}_{\mathrm{C} 1}$ films.

The amount of PW in the $\mathrm{PCH}$ films was determined by thermogravimetric analysis (TGA, Figure 2d). The results were in good agreement with the direct weight measurements of the pristine and hybrid films. For example, the weight percentage of $\mathrm{OD}_{\mathrm{H}}$ was determined to be $50.3 \%$ from TGA, which matched the difference between the pristine $(5.2 \mathrm{mg})$ and $\mathrm{OD}_{\mathrm{H}}-\mathrm{PDPA}_{\mathrm{C} 1}(10.3 \mathrm{mg})$ films. The weight percentages of $\mathrm{HD}_{\mathrm{H}}, \mathrm{IS}_{\mathrm{H}}, \mathrm{OD}_{\mathrm{OH}}$ and $\mathrm{OD}_{\mathrm{COOH}}$ in the $\mathrm{PCH}$ films were determined to be $51.2 \%, 53.7 \%, 42.4 \%$ and $38.4 \%$, respectively, based on TGA (Table 1). A cross-sectional scanning electron microscope image of a PCH film demonstrated that the $\mathrm{PW}$ is completely incorporated in $\mathrm{PDPA}_{\mathrm{C} 1}$, with no apparent discontinuities (Supplementary Figure 3). The PDPA $\mathrm{C}_{1}$ microvoids appear to take up PW collectively on contact. Notably, all of the $\mathrm{PCH}$ films remained stable without measureable PW leakage, even when held at elevated temperatures continuously for 1 week. The $\mathrm{PCH}$ films did not undergo any significant changes in weight or structural features even after the prolonged heating (Supplementary Figure 4) because the high miscibility between the $\mathrm{PDPA}_{\mathrm{C} 1}$ and $\mathrm{PWs}$ can help in maintaining close contact during the phase change.

The X-ray diffraction patterns reveal the conformational and structural changes experienced by the matrix polymer within our $\mathrm{PCH}$ films (Figure 2e). The pristine film produced a sharp signal due to the lamellar layer at a small angle of $7.1^{\circ}$ (interlayer distance $\approx 12.4 \AA$ according to the Bragg equation). The diffraction peak shifted to a lower angle in the hybrid films. The small-angle peaks appeared at $5.6^{\circ}$ $\left(\mathrm{HD}_{\mathrm{H}}\right), 6.2^{\circ}\left(\mathrm{OD}_{\mathrm{H}}\right), 6.4^{\circ}\left(\mathrm{IS}_{\mathrm{H}}\right), 6.5^{\circ}\left(\mathrm{OD}_{\mathrm{COOH}}\right)$ and $6.8^{\circ}\left(\mathrm{OD}_{\mathrm{OH}}\right)$, depending on the type of PW. Shifts to the lower-angle side suggested that the lamellar layer distance increased in the hybrids because of the plasticizer effect of the PWs. The degree of lower-angle shift decreased as the molecular weight or polarity of the PWs increased. Broad scattering signals were also observed at wide angles. The pristine film produced a single peak at approximately $25^{\circ}(3.5 \AA)$, whereas an additional new signal appeared at approximately $18.5^{\circ}(4.8 \AA)$ for the hybrid films. A distance of $3.5 \AA$ is common for $\pi$-stacking supramolecular interactions, whereas a distance of $4.8 \AA$ is typical of a disordered, amorphous structure. ${ }^{34}$ Therefore, the broad peak at $25^{\circ}$ must be assigned to the intramolecular stack structure of the side phenyl rings, whereas the peak at $18.5^{\circ}$ is due to the PWs $\left(\mathrm{HD}_{\mathrm{H}}\right.$, $\left.\mathrm{OD}_{\mathrm{H}}\right)$, which are in the molten state at ambient temperature. We surmise that the ordered structures in the pristine $\mathrm{PDPA}_{\mathrm{C} 1}$ film collapsed upon hybridization with the PWs, whereas the intramolecular stack structure simultaneously underwent relaxation. These conformational and structural changes in the $\mathrm{PDPA}_{\mathrm{C} 1}$ matrix should be responsible for the FL emission enhancement and blue shift in the hybrid film. Additionally, the hybrid films containing high-melting PWs $\left(\mathrm{OD}_{\mathrm{OH}}, \mathrm{OD}_{\mathrm{COOH}}\right.$ and $\left.\mathrm{IS}_{\mathrm{H}}\right)$ produced sharp peaks over a wide angle range. These features were attributed to the PWs crystallized at ambient temperature.

The latent heat storage/release behaviors of the $\mathrm{PCH}$ films were evaluated using DSC. Figure 3a presents the DSC curves of the $\mathrm{PCH}$ films. During heating, the $\mathrm{OD}_{\mathrm{H}}-\mathrm{PDPA}_{\mathrm{C} 1}$ system exhibited a thermodynamic $T_{m}$ at $29.8^{\circ} \mathrm{C}$ due to an endothermic volume change in $\mathrm{OD}_{\mathrm{H}}$. The pristine film exhibited neither a phase transition nor local molecular relaxation in this temperature range. During cooling, the hybrid apparently underwent exothermic crystallization $\left(T_{c}\right)$ of $\mathrm{OD}_{\mathrm{H}}$ at $22.9^{\circ} \mathrm{C}$. The endothermic peak shifted to a lower temperature by $2{ }^{\circ} \mathrm{C}$ compared with that of pure $\mathrm{OD}_{\mathrm{H}}$ (Table 1 , Figure $3 \mathrm{~b}$ ). The absolute enthalpy $\left(\Delta H_{f u s, a b s}\right)$ for $\mathrm{OD}_{\mathrm{H}}$ in the hybrid film, obtained by dividing the apparent enthalpy $\left(\Delta H_{f u s}\right.$, including the mass of PDPA $\left.\mathrm{C}_{\mathrm{C} 1}\right)$ by the $\mathrm{OD}_{\mathrm{H}}$ loading, was determined to be $131.9 \mathrm{Jg}^{-1}$ (Table 1). The $\Delta H_{f u s, a b s}$ value of $\mathrm{OD}_{\mathrm{H}}$ in the hybrids was $\sim 74 \%$ of that in the pure PW (Table 1). Similar trends were observed for the other hybrid films in their thermodynamic behaviors (Figure 3a). The $\Delta H_{f u s, ~ a b s}\left(T_{m}\right)$ values of the $\mathrm{HD}_{\mathrm{H}}-, \mathrm{IS}_{\mathrm{H}}-, \mathrm{OD}_{\mathrm{OH}}-$ and $\mathrm{OD}_{\mathrm{COOH}}-\mathrm{PDPA}_{\mathrm{C} 1}$ hybrid films were $159.7\left(20.1{ }^{\circ} \mathrm{C}\right), 111.4\left(37.6^{\circ} \mathrm{C}\right), 60.1\left(59.1^{\circ} \mathrm{C}\right)$ and 31.8 $\left(69.7^{\circ} \mathrm{C}\right) \mathrm{J} \mathrm{g}^{-1}$, respectively. These values are approximately $87 \%, 55 \%$, $39 \%$ and $30 \%$ of those of the corresponding pure PWs, respectively (Table 1). The relative enthalpy of the hybrid films decreased as the molecular weight and polarity of the PW increased. The lower $T_{m}$ and $\Delta H_{f u s, a b s}$ of the hybrids relative to those of the pure PWs are most likely due to the portion of PW molecules that is randomly arranged in the amorphous domain of $\mathrm{PDPA}_{\mathrm{C} 1}$. Notably, the $\Delta H_{f u s, a b s}$ of the hybrid films remained constant during several repeated heating and cooling processes, confirming that the $\mathrm{PWs}$ within the $\mathrm{PDPA}_{\mathrm{C} 1}$ matrix exist in a stable form (Figure 3c, Supplementary Figure 5).

A covalently $\mathrm{OD}_{\mathrm{H}}-$-coupled $\mathrm{PDPA}$ derivative $\left(\mathrm{PDPA}_{\mathrm{C} 18}\right.$ in Figure 1) synthesized in a previous study ${ }^{34,35}$ exhibited an endothermic transition at a much lower temperature of $-1{ }^{\circ} \mathrm{C}$, with a very low 

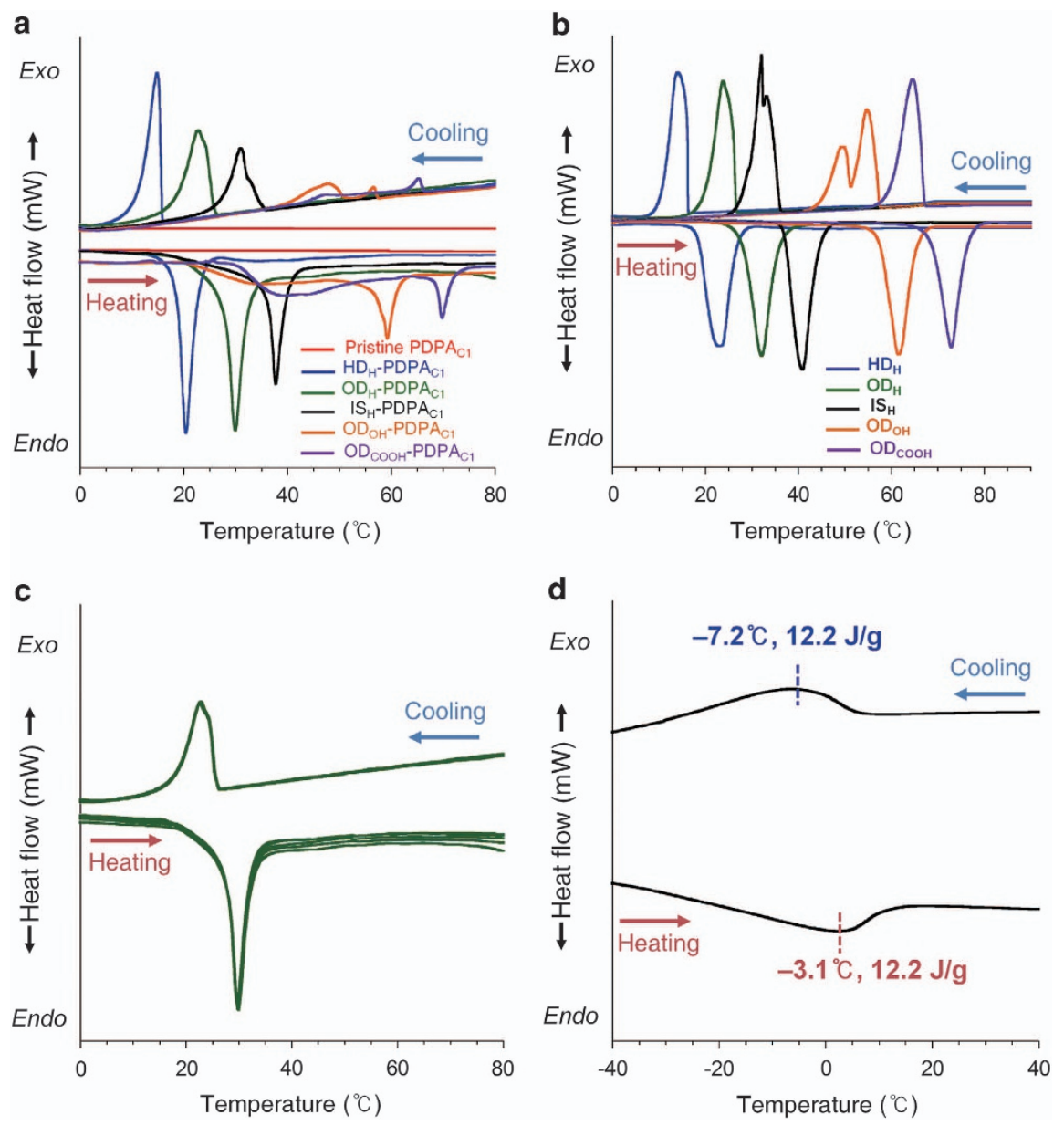

Figure 3 DSC thermograms. (a) $\mathrm{PCH}$ films, (b) pure $\mathrm{PWs,} \mathrm{(c)} \mathrm{OD}_{\mathrm{H}}-\mathrm{PDPA}_{\mathrm{C} 1}$ films during 5 cycles, (d) $\mathrm{PDPA}_{\mathrm{c} 18}$ (heat flow rate $\approx 1$ min ${ }^{\circ} \mathrm{C}^{-1}$, under nitrogen gas).

$\Delta H_{f u s, ~ a b s}$ of $12.3 \mathrm{~J} \mathrm{~g}^{-1}$ (Figure 3d). This finding most likely results from the side $\mathrm{OD}_{\mathrm{H}}$ groups being covalently attached to the highly twisted backbone, leading to significantly weakened intermolecular forces, a longer intermolecular distance and a disordered structure between the side alkyl chains. This result suggests that the $\mathrm{OD}_{\mathrm{H}}-\mathrm{PDPA}_{\mathrm{C} 1}$ hybrid system is more suitable than the single-component $\mathrm{PDPA}_{\mathrm{C} 18}$ system for efficient heat storage.

The $\mathrm{PCH}$ films provided a unique optical signal in the heat storage/release process. Variations in the FL emission spectra of the $\mathrm{OD}_{\mathrm{H}}-\mathrm{PDPA}_{\mathrm{C} 1}$ hybrid are shown in Figures $4 \mathrm{a}$ and $\mathrm{b}$ under heating and cooling over the temperature range of $10-35^{\circ} \mathrm{C}$ (rates of $20^{\circ} \mathrm{C} \mathrm{min}-1$ ). During the endothermic volume expansion of $\mathrm{OD}_{\mathrm{H}}$ upon heating from 27.5 to $30^{\circ} \mathrm{C}$, the $\mathrm{FL}$ emission of the $\mathrm{OD}_{\mathrm{H}}-\mathrm{PDPA}_{\mathrm{C} 1}$ hybrid film was sharply attenuated (the intensity decreased to one-half of the initial), with a notable color change (from yellow to green due to the new shoulder band at $475 \mathrm{~nm}$ ) (Figure 4a). An opposite change was observed during the exothermic volume contraction upon cooling from $22.5^{\circ} \mathrm{C}$ down to $17.5^{\circ} \mathrm{C}$ (Figure $4 \mathrm{~b}$ ); a drastic FL enhancement (doubling of intensity) and color change (from green to yellow due to the disappearance of the shoulder at $475 \mathrm{~nm}$ ) could be observed. The shorter-wavelength band at $475 \mathrm{~nm}$ is due to the kinked structure of the PDPA $\mathrm{C} 1_{1}$ chains in the swollen state. ${ }^{34}$ This critical variation was entirely reversible upon repeated temperature cycling (Figure 4c). The temperature range over which FL changes were observed was in accordance with the phase-change temperature observed in DSC (Figure 4d). The pristine film lacked these properties (Supplementary Figure 6).

The unique FL response of our PCH films to latent heat enabled their application in a write/erase sensor system for imaging and patterning (Figure 5). When a fully cooled $\mathrm{OD}_{\mathrm{H}}-\mathrm{PDPA}_{\mathrm{C} 1}$ hybrid film was held at $25^{\circ} \mathrm{C}$ over the entire region and then brought into contact with a warm metal rod (stainless-steel spatula in this study), apparent FL characters could be written on the film as a turn-off system (Figure 5a, Supplementary Movie 1). Subsequently, when the film was cooled again over the same area, the FL character images could be erased. In contrast, when a fully warmed $\mathrm{OD}_{\mathrm{H}}-\mathrm{PDPA}_{\mathrm{C} 1}$ film held at $27^{\circ} \mathrm{C}$ was touched with a cooled spatula, distinctive character images were obtained as a turn-on mode (Figure 5a, Supplementary Movie 2). The images could subsequently be erased by re-heating the film over the entire region.

The temperature of the human body is maintained near $36.5^{\circ} \mathrm{C}$ under conditions of normal health, and this value is significantly higher than the $T_{\mathrm{m}}\left(32.0^{\circ} \mathrm{C}\right)$ of $\mathrm{OD}_{\mathrm{H}}$. Hence, the $\mathrm{OD}_{\mathrm{H}}-\mathrm{PDPA}_{\mathrm{C} 1}$ hybrids could potentially be used in a diverse range of sensor applications. When the hybrid films were below $25^{\circ} \mathrm{C}$ and brought into contact with the human body, the FL emission was immediately quenched because of the melting of $\mathrm{OD}_{\mathrm{H}}$. In particular, if the film was touched softly with a finger, a high-resolution FL image of the fingerprint could be observed under a standard ultraviolet lamp light (Figure 5b, Supplementary Movie 3). The fingerprint image was then readily erased by a heating/cooling cycle, and the film could be reused. 
a

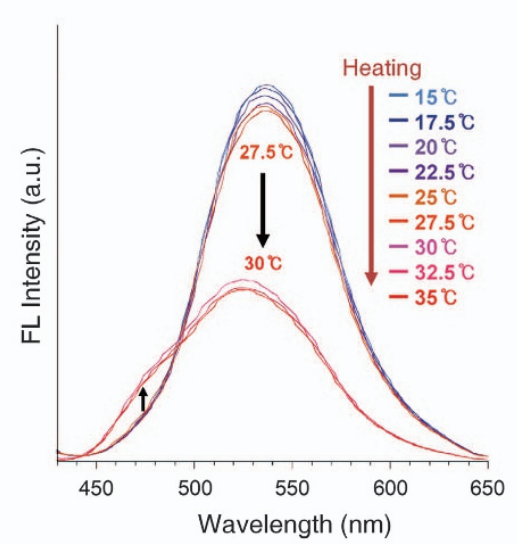

C

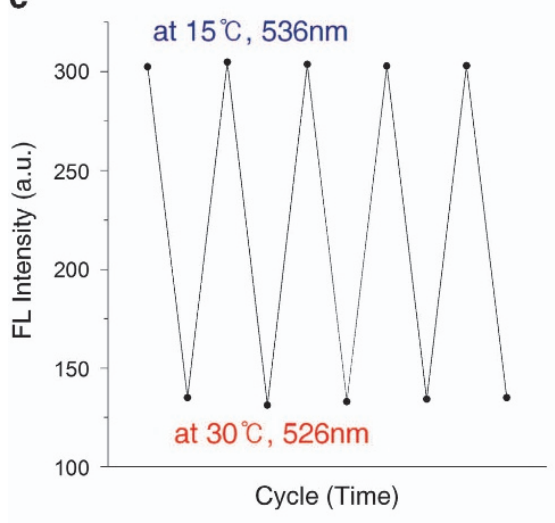

b

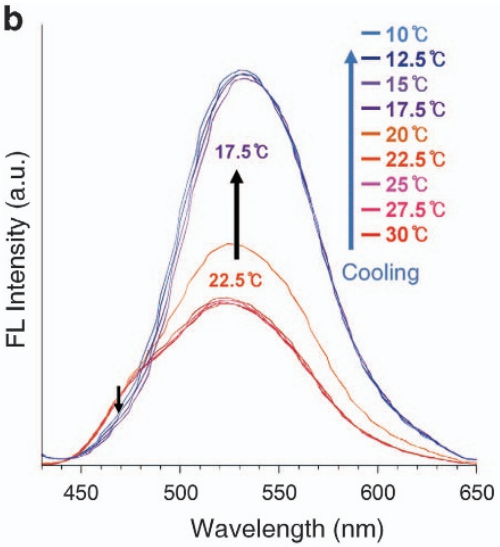

d

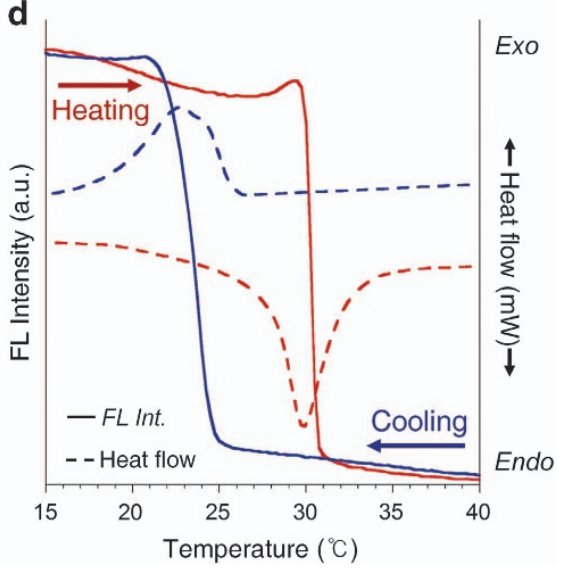

Figure $4 \mathrm{FL}$ emission spectra. Variation in $\mathrm{FL}$ emission spectra of $\mathrm{OD}_{\mathrm{H}}-\mathrm{PDPA}_{\mathrm{C1}}$ film upon (a) heating and (b) cooling (excited at $420 \mathrm{~nm}$, heat flow rate $\approx 20^{\circ} \mathrm{C} \mathrm{min}{ }^{-1}$ ). (c) FL intensity of $O D_{H}-P D P A_{\mathrm{C} 1}$ film during five heating (at $30^{\circ} \mathrm{C}, 526 \mathrm{~nm}$ ) and cooling (at $15^{\circ} \mathrm{C}, 536 \mathrm{~nm}$ ) cycles (excited at $420 \mathrm{~nm}$, monitored at maximum intensity wavelength). (d) Comparative variation in $\mathrm{FL}$ intensity and heat flow of $\mathrm{OD}_{\mathrm{H}}-\mathrm{PDPA}_{\mathrm{Cl}}$ film upon heating and cooling (excited at $420 \mathrm{~nm}$, monitored at $560 \mathrm{~nm}$ ).

a
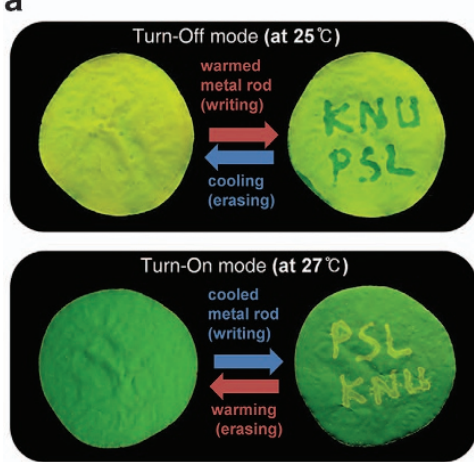

b

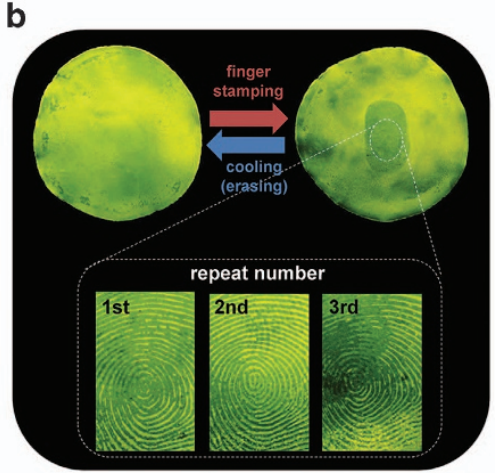

C

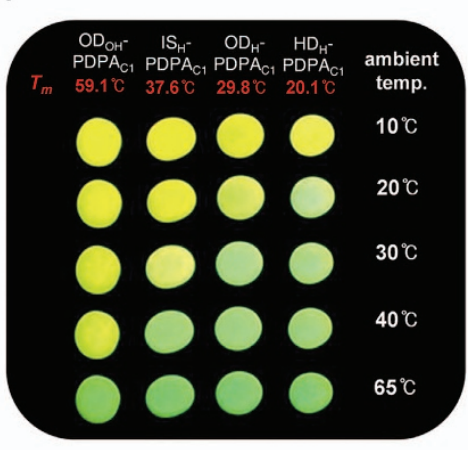

Figure 5 Sensor applications. Temperature-responsive writing/erasing system for (a) FL imaging and (b) fingerprinting (a freestanding $O D_{H}-P_{D P A} A_{1}$ hybrid film with thickness $>100 \mu \mathrm{m}$ was used and excited at $>365 \mathrm{~nm}$ ). (c) Thermosensor array system (four spots were arrayed on the same glass slide by simply casting four drops of PDPA $\mathrm{Cl}_{1}$ solution and subsequently depositing PW on each spot).

This observation suggests the potential applicability of the hybrid films in fingerprint recognition systems. The present materials hint at a completely new approach relative to conventional reflectance-, capacitance- or ultrasound-based fingerprint sensors. ${ }^{49}$ Moreover, when various $\mathrm{PWs}\left(\mathrm{HD}_{\mathrm{H}}, \mathrm{OD}_{\mathrm{H}}, \mathrm{IS}_{\mathrm{H}}, \mathrm{OD}_{\mathrm{OH}}\right)$ with different $T_{m}$ values were deposited on the $\mathrm{PDPA}_{\mathrm{C} 1}$ film as a platform sensor array, more accurate information about the ambient temperature was obtained because each $\mathrm{PW}-\mathrm{PDPA}_{\mathrm{C} 1}$ location has a different critical FL evolution temperature (Figure 5c, Supplementary Movie 4). The present system may be used as an FL thermometer for medical examination and for air-conditioning applications. ${ }^{50-53}$

BOPP films, which are commercialized as tobacco packaging materials, are known to be exceptionally tough, flexible, transparent and chemical-repellent. These physical properties meet all the requirements for a supporting layer in the actuator applications suggested by our study. ${ }^{33}$ A bilayer film was prepared by casting the $\mathrm{PDPA}_{\mathrm{C} 1}$ solution in toluene onto a BOPP film and then infiltrating the polymer bilayers with $\mathrm{OD}_{\mathrm{H}}$ at $35^{\circ} \mathrm{C}$ (Figure 6a). The $\mathrm{OD}_{\mathrm{H}^{-}}$ 
impregnating bilayer films became flexible, tough and mechanically robust because the $\mathrm{PDPA}_{\mathrm{C} 1}$ is in intimate contact with an underlying BOPP layer with similar surface properties. Figure $6 \mathrm{~b}$ and Supplementary Movie 5 illustrate the thermomechanical actuation of the bilayer film. Curling occurred immediately when cooling was introduced using a dry ice pack. The film uncurled completely, returning to its initial state when the dry ice pack was removed. Only a few seconds were required for the films to reach the minimum radius of curvature upon cooling. Uncurling back to almost $180^{\circ}$ (radius of curvature $\approx \infty$ ) was virtually instantaneous upon warming at room temperature.

\section{DISCUSSION}

To explain the unusual FL emission changes observed in Figure 4, it is necessary to consider the molecular thermodynamics of the PWs surrounding the $\mathrm{PDPA}_{\mathrm{C} 1}$ matrix. In a previous study, we observed that the FL intensity of $\mathrm{PDPA}_{\mathrm{C} 1}$ varied significantly depending on the

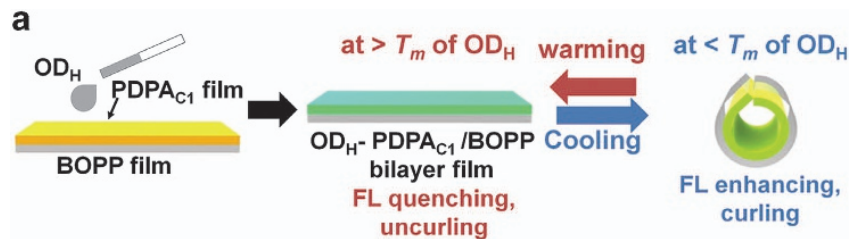

b

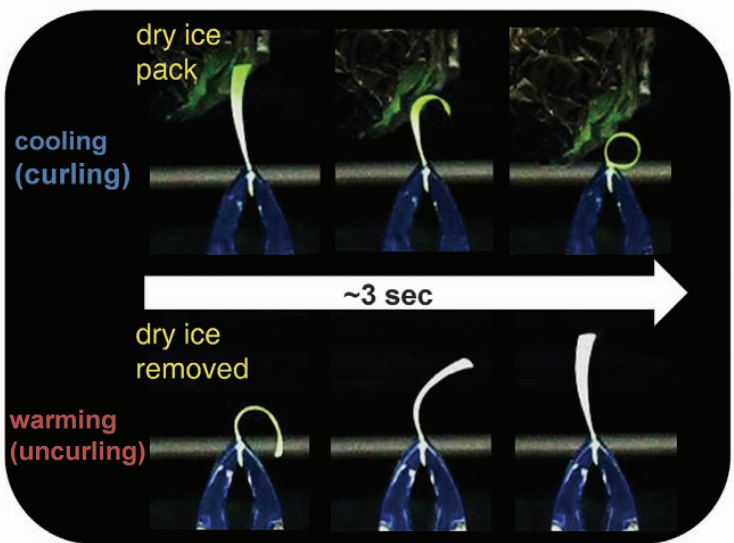

C

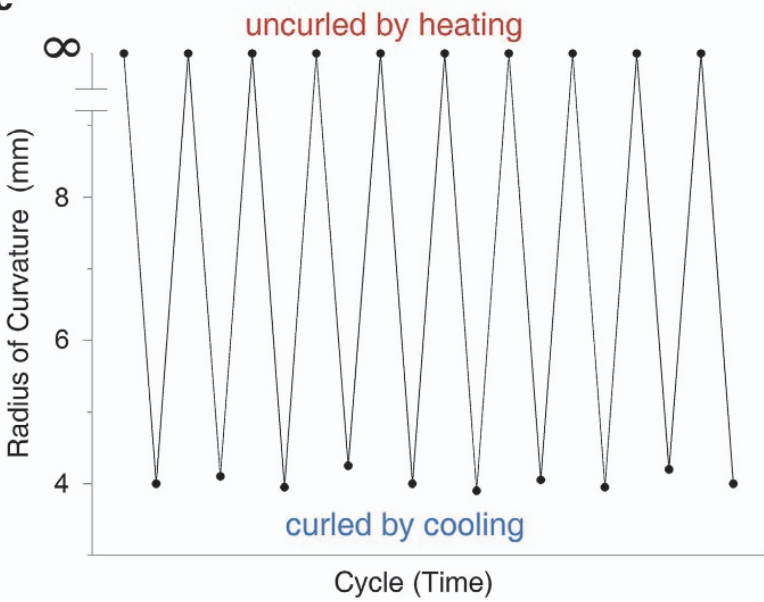

Figure 6 Actuator applications. (a) Schematic illustration and (b) FL images of the $\mathrm{OD}_{\mathrm{H}}-\mathrm{PDPA}_{\mathrm{C} 1} / \mathrm{BOPP}$ bilayer film. (c) Radius of curvature during 10 heating and cooling cycles (conducted at approximately $40^{\circ} \mathrm{C}$ and $10^{\circ} \mathrm{C}$ ). viscosity of the solvent in the swollen state because the degree of collisional quenching depends on the viscosity of the medium. ${ }^{37}$ That is, the molecular friction energy transferred from the medium to the $\mathrm{PDPA}_{\mathrm{C} 1}$ matrix should increase with a decrease in the viscosity of the medium, leading to greater perturbation of the polymer chains. In other words, the side phenyl rings should experience significant molecular perturbation during melting of the PWs because the crystal-to-liquid phase transition will greatly increase the collision frequencies between the polymer chains and the PWs. Accordingly, $\mathrm{PDPA}_{\mathrm{C} 1}$ should undergo nonradiative emission decay due to collisional quenching and vibrational relaxation. Under cooling, the opposing thermodynamic behavior (a liquid-to-crystal transition) of the PWs should be responsible for the critical FL increase.

The reversible actuation could be repeated almost indefinitely and was highly reproducible (Figure $6 \mathrm{c}$ ). No signs of mechanical fatigue could be observed. Moreover, the actuator film immediately reverted to its initial configuration when brought in contact with the human body because of the body heat (Supplementary Figure 7). The curling behavior is attributable to the exothermic volume contraction associated with $\mathrm{OD}_{\mathrm{H}}$ crystallization. The uncurling under warming is due to the endothermic volume expansion accompanying the crystal-to-liquid phase transition. The volume change of pure $\mathrm{OD}_{\mathrm{H}}$ under melting is documented to be $19.7 \% .{ }^{54}$ Our $\mathrm{OD}_{\mathrm{H}}-\mathrm{PDPA}_{\mathrm{C} 1}$ hybrid film was approximately $207 \mu \mathrm{m}$ thick when cooled to $10^{\circ} \mathrm{C}$ but swelled to $237 \mu \mathrm{m}$ at $30^{\circ} \mathrm{C}$, representing a volume increase of $14.5 \%$ (Supplementary Figure 8). The tightening and relaxation movements, in turn, can be ascribed to the interfacial stress induced by a large mismatch in volume changes for the active $\mathrm{OD}_{\mathrm{H}}-\mathrm{PDPA}_{\mathrm{C} 1}$ hybrid and inactive BOPP layers.

In summary, we developed multifunctional fully organic PCHs that can potentially be applied to next-generation sensor-actuator technologies. Unique $\mathrm{PW}-\mathrm{PDPA}_{\mathrm{C} 1}$ hybrid films were obtained by infiltrating $\mathrm{PDPA}_{\mathrm{C} 1}$ films with liquid-state PWs. The resultant $\mathrm{PCH}$ s exhibited remarkable FL changes and mechanical deformation properties during latent heat storage/release processes. The phase-change enthalpy of $\mathrm{PW}$ in our PCH films was quite high and remained constant over a long period under test usage, with no apparent leakage. Both the FL response and the thermomechanical actuation were rapid and highly reproducible. Our findings should be helpful for developing novel functional PCH materials. Various combinations of organic PCMs and $\mathrm{CPs}$ in hybrid systems are expected to find further applications.

\section{CONFLICT OF INTEREST}

The authors declare no conflict of interest.

\section{ACKNOWLEDGEMENTS}

This study was supported by the Basic Science Research Program through the National Research Foundation of Korea (NRF) grant funded by the Korean government (MEST) (2008-0062617). This research was also supported by a grant from the R\&D Program (Nanotechnology Convergence Project for Commercialization and Platform, Grant No. 10036099) funded by the Ministry of Knowledge Economy (MKE), Republic of Korea. In addition, the authors are deeply thankful to all interested persons of MKE and KEIT (Korea Evaluation Institute of Industrial Technology). The New \& Renewable Energy Core Technology Program of the Korea Institute of Energy Technology Evaluation and Planning (KETEP) provided financial resource from the Ministry of Trade, Industry \& Energy, Republic of Korea (No. 20133030000210).

Author contributions: Y-JJ designed the project and performed the majority of the experiments and analysis of the data. Y-JJ, GK and S-YJ prepared the manuscript for the paper. BS-IK, W-EL, C-LL, HK and K-HS contributed analysis tools. GK and S-YJ directed the project. All the authors discussed the results and contributed to the paper. 
$1 \mathrm{Ge}, \mathrm{H}$., Li, H., Mei, S. \& Liu, J. Low melting point liquid metal as a new class of phase change material: An emerging frontier in energy area. Renewable Sustainable Energy Rev 21, 331-346 (2013).

2 Sarier, N. \& Onder, E. Organic phase change materials and their textile applications: An overview. Thermochim. Acta 540, 7-60 (2012).

3 Zhou, D., Zhao, C. Y. \& Tian, Y. Review on thermal energy storage with phase change materials (PCMs) in building applications. Appl. Energy 92, 593-605 (2012).

4 Oro, E., Gracia, A. D., Castell, A., Farid, M. M. \& Cabeza, L. F. Review on phase change materials (PCMs) for cold thermal energy storage applications. Appl. Energy 99 513-533 (2012).

5 Kuznik, F., David, D., Johannes, K. \& Roux, J.-J. A review on phase change materials integrated in building walls. Renewable Sustainable Energy Rev. 15, 379-391 (2010).

6 Li, B., Liu, T., Hu, L., Wang, Y. \& Gao, L. Fabrication and properties of microencapsulated paraffin@SiO2 phase change composite for thermal energy storage. ACS Sustainable Chem. Eng 1, 374-380 (2013).

7 Chen, L., Zou, R, Xia, W., Liu, Z., Shang, Y., Zhu, J., Wang, Y., Lin, J., Xia, D. \& Cao, A. Electro- and photodriven phase change composites based on wax-infiltrated carbon nanotube sponges. ACS Nano. 6, 10884-10892 (2012).

8 Wang, Y., Tang, B. \& Zhang, S. Novel organic solar thermal energy storage materials: efficient visible light-driven reversible solid-liquid phase transition. J. Mater. Chem. 22 18145-18150 (2012)

9 Wu, S., Zhu, D., Zhang, X. \& Huang, J. Preparation and melting/freezing characteristics of Cu/paraffin nanofluid as phase-change material (PCM). Energy Fuels 24, 1894-1898 (2010).

10 Hong, Y., Ding, S., Wu, W., Hu, J., Voevodin, A. A., Gschwender, L., Snyder, E., Chow, L. \& $\mathrm{Su}, \mathrm{M}$. Enhancing heat capacity of colloidal suspension using nanoscale encapsulated phasechange materials for heat transfer. ACS Appl. Mater. Interfaces 2, 1685-1691 (2010).

11 Golemanov, K., Tcholakova, S., Denkov, N. D. \& Gurkov, T. Selection of surfactants for stable paraffin-in-water dispersions, undergoing solid-liquid transition of the dispersed particles. Langmuir 22, 3560-3569 (2006).

12 Ogden, S., Klintberg, L., Thornell, G., Hjort, K. \& Boden, R. Review on miniaturized paraffin phase change actuators. Microfluid Nanofluid 17, 53-71 (2014).

13 Park, J.-M., Cho, Y.-K., Lee, B.-S., Lee, J.-G. \& Ko, C. Multifunctional microvalves control by optical illumination on nanoheaters and its application in centrifugal microfluidic devices. Lab Chip 7, 557-564 (2007).

14 Abi-Samra, K., Hanson, R., Madou, M. \& Gorkin, R. A. III Infrared controlled waxes for liquid handling and storage on a CD-microfluidic platform. Lab Chip 11 723-726 (2011).

15 Thomas, S. W. III., Joly, G. D. \& Swager, T. M. Chemical sensors based on amplifying fluorescent conjugated polymers. Chem. Rev. 107, 1339-1386 (2007).

16 McQuade, D. T., Pullen, A. E. \& Swager, T. M. Conjugated polymer-based chemical sensors. Chem. Rev. 100, 2537-2574 (2000).

17 Swager, T. M. The molecular wire approach to sensory signal amplification. Acc. Chem. Res. 31, 201-207 (1998).

18 Smela, E. Conjugated polymer actuators. MRS Bull. 33, 197-204 (2008).

19 Jager, E. W. H., Smela, E. \& Inganas, O. Microfabricating conjugated polymer actuators Science 290, 1540-1546 (2000).

20 Bunz, U. H. F. Poly(aryleneethynylene)s. Macromol. Rapid Commun. 30, 772-805 (2009)

21 Grenier, C. R., Pisula, W., Joncheray, T. J., Muller, K. \& Reynolds, J. R. Regiosymmetric poly(dialkylphenylenedioxythiophene)s: electron-rich, stackable $\pi$-conjugated nanoribbons. Angew. Chem. Int. Ed. 46, 714-717 (2007).

22 Taylor, M. S. \& Swager, T. M. Poly(anthrylenebutadiynylene)s: precursor-based synthesis and band-gap tuning. Angew. Chem. Int. Ed. 46, 8480-8483 (2007).

23 Bunz, U. H. F. Poly(aryleneethynylene)s: syntheses, properties, structures, and applications. Chem. Rev. 100, 1605-1644 (2000).

24 Kraft, A., Grimsdale, A. C. \& Homes, A. B. Electroluminescent conjugated polymersseeing polymers in a new light. Angew. Chem. Int. Ed. 37, 403-428 (1998).

25 Cornil, J., dos Santos, D. A., Crispin, X., Silbey, R. \& Bredas, J. L. Influence of interchain interactions on the absorption and luminescence of conjugated oligomers and polymers: a quantum-chemical characterization. J. Am. Chem. Soc. 120, 1289-1229 (1998)

26 Kanaya, T., Tsukushi, I., Kaji, K., Sakaguchi, T., Kwak, G. \& Masuda, T. Role of local dynamics in the gas permeability of glassy substituted polyacetylenes. a quasielastic neutron scattering study. Macromolecules 35, 5559-5564 (2002).

27 Toy, L. G., Nagai, K., Freeman, B. D., Pinnau, I., He, Z., Masuda, T., Teraguchi, M. \& Yampolskii, Y. P. Pure-gas and vapor permeation and sorption properties of poly[1-phenyl-2-[p-(trimethylsilyl)phenyl]acetylene] (PTMSDPA). Macromolecules 33, 2516-2524 (2000).

28 Park, H., Han, D.-C., Han, D.-H., Kim, S.-J., Lee, W.-E. \& Kwak, G. Emission enhancement, photooxidative stability, and fluorescence image patterning of conjugated polymer film via in situ hybridization with UV-curable acrylate monomers. Macromolecules 44, 9351-9355 (2011).

29 Park, H., Jeong, H., Lee, W.-E., Yoon, K.-B., Oh, C.-J. \& Kwak, G. Positive-/negativeerasable-/immobilized-, mono-/multi-color fluorescence image patterning of molecularscale porous polymer film via a microcontact printing method using various chemical inks. Macromol. Rapid Commun. 32, 360-365 (2011).

30 Jeong, H., Lee, W.-E. \& Kwak, G. Enhancements in emission and chemical resistance of substituted acetylene polymer via in situ sol-gel reaction in film. Macromolecules 43, 1152-1155 (2010)
31 Kwak, G., Lee, W.-E., Jeong, H., Sakaguchi, T. \& Fujiki, M. Swelling-induced emission enhancement in substituted acetylene polymer film with large fractional free volume: fluorescence response to organic solvent stimuli. Macromolecules 42, 20-24 (2009).

32 Kwak, G., Lee, W.-E., Kim, W.-H. \& Lee, H. Fluorescence imaging of latent fingerprints on conjugated polymer films with large fractional free volume. Chem. Commun. 2112-2114 (2009)

33 Lee, W.-E., Jin, Y.-J., Park, L.-S. \& Kwak, G. Fluorescent actuator based on microporous conjugated polymer with intramolecular stack structure. Adv. Mater. 24 5604-5609 (2012).

34 Lee, W.-E., Kim, J. W., Oh, C. J., Sakaguchi, T., Fujiki, M. \& Kwak, G. Correlation of intramolecular excimer emission with lamellar layer distance in liquid-crystalline polymers: verification by the film-swelling method. Angew. Chem. Int. Ed. 49, 1406-1409 (2010)

35 Jin, Y.-J., Bae, J.-E., Cho, K.-S., Lee, W.-E., Hwang, D.-Y. \& Kwak, G. Room temperature fluorescent conjugated polymer gums. Adv. Funct. Mater. 24, 1928-1932 (2014).

36 Kim, S.-I., Jin, Y.-J., Lee, W.-E., Yu, R., Park, S.-J., Kim, H.-J., Song, K.-H. \& Kwak, G. Microporous conjugated polymers with enhanced emission in immiscible two-phase system in response to surfactants. Adv. Mater. Interfaces 1, 1300029 (2014).

37 Lee, W.-E., Lee, C.-L., Sakaguchi, T., Fujiki, M. \& Kwak, G. Fluorescent viscosity sensor film of molecular-scale porous polymer with intramolecular $\pi$-stack structure. Macromolecules 44, 432-436 (2011).

$38 \mathrm{Ohm}$, C., Brehmer, M. \& Zentel, R. Liquid crystalline elastomers as actuators and sensors. Adv. Mater. 22, 3366-3387 (2010).

39 Yu, Y. \& Ikeda, T. Soft actuators based on liquid-crystalline elastomers. Angew. Chem. Int. Ed 45, 5416-5418 (2006).

40 Wu, W., Yao, L., Yang, T., Yin, R., Li, F. \& Yu, Y. NIR-light-induced deformation of cross-linked liquid-crystal polymers using upconversion nanophosphors. J. Am. Chem. Soc. 133, 15810-15813 (2011).

41 Jiang, Z., Xu, M., Li, F. \& Yu, Y. J. Red-light-controllable liquid-crystal soft actuators via low-power excited upconversion based on triplet-triplet annihilation. Am. Chem. Soc 135, 16446-16453 (2013).

42 Kohlmeyer, R. R. \& Chen, J. Wavelength-selective IR light-driven hinges based on liquid crystalline elastomer composites. Angew. Chem. Int. Ed. 52, 9234-9237 (2013)

43 de Haan, L. T., Sanchez-Somolinos, C., Bastiaansen, C. M. W., Schenning, A. P. H. J. \& Broer, D. J. Engineering of complex order and the macroscopic deformation of liquid crystal polymer networks. Angew. Chem. Int. Ed. 51, 12469-12472 (2012).

44 Tsuchihara, K., Masuda, T. \& Higashimura, T. Tractable silicon-containing poly (diphenylacetylenes): their synthesis and high gas permeability. J. Am. Chem. Soc 113, 8548-8549 (1991).

45 Sakaguchi, T., Yumoto, K., Shiotsuki, M., Sanda, F., Yoshikawa, M. \& Masuda, T. Synthesis of poly(diphenylacetylene) membranes by desilylation of various precursor polymers and their properties. Macromolecules 38, 2704-2709 (2005).

46 Lee, W.-E., Han, D. C., Han, D. H., Choi, H. J., Sakaguchi, T., Lee, C. L. \& Kwak, G Remarkable change in fluorescence emission of poly(diphenylacetylene) film via in situ desilylation reaction: correlation with variations in microporous structure, chain conformation, and lamellar layer distance. Macromol. Rapid Commun. 32, 1047-1051 (2011).

47 Barbillon, P., Schuffenecker, L., Dellacherie, J., Balesdent, D. \& Dirande, M. Enthalpy change from 260 to $340 \mathrm{~K}$ for all normal paraffins from octadecane (n-C18) to hexacosane (n-C26). J. Chim. Phys. Phys.-Chim. Biol. 88, 91-113 (1991).

48 Parks, G. S., Moore, G. E., Renquist, M. L., Naylor, B. F., McClaine, L. A., Fujii, P. S. \& Hatton, J. A. Thermal data on organic compounds. XXV. Some heat-capacity, entropy, and free-energy data for nine hydrocarbons of high molecular weight. J. Am. Chem. Soc 71, 3386-3389 (1949)

49 Maltoni, D., Maio, D., Jain, A. K. \& Prabhakar, S. Handbook of Fingerprint Recognition 2nd edn Springer, (2009).

50 Yan, D., Lu, J., Ma, J., Wei, M., Evans, D. G. \& Duan, X. Reversibly thermochromic, fluorescent ultrathin films with a supramolecular architecture. Angew. Chem. Int. Ed. 50, 720-723 (2011).

51 Feng, J., Tian, K., Hu, D., Wang, S., Li, S., Zeng, Y., Li, Y. \& Yang, G. A triarylboronbased fluorescent thermometer: sensitive over a wide temperature range. Angew. Chem. Int. Ed. 50, 8072-8076 (2011).

52 Cauzzi, D., Pattacini, R., Delferro, M., Dini, F., Di Natale, C., Paolesse, R., Bonacchi, S., Montalti, M., Zaccheroni, N., Calvaresi, M., Zerbetto, F.- \&, Prodi, L. Temperaturedependent fluorescence of Cu5 metal clusters: a molecular thermometer. Angew. Chem. Int. Ed. 51, 9662-9665 (2012).

53 Sun, X., Zhang, Z., Lu, X., Guan, G., Li, H. \& Peng, H. Electric current test paper based on conjugated polymers and aligned carbon nanotubes. Angew. Chem. Int. Ed. 52, 7776-7780 (2013)

54 Muller, A. \& Lonsdale, K. The low-temperature form of octadecane. Acta Cryst 1 129-131 (1948).

(c) (i) This work is licensed under a Creative Commons Attribution 3.0 Unported License. The images or other third party material in this article are included in the article's Creative Commons license, unless indicated otherwise in the credit line; if the material is not included under the Creative Commons license, users will need to obtain permission from the license holder to reproduce the material. To view a copy of this license, visit http:// creativecommons.org/licenses/by/3.0/

Supplementary Information accompanies the paper on the NPG Asia Materials website (http://www.nature.com/am) 